\title{
Giving the Nod to Bacterial Symbionts: The Nod Signal Transduction Pathway
}

\author{
Kevin Murray
}

\section{Abstract}

This in-depth review summarises recent advances in the elucidation of the Nod factor-induced signal transduction pathway. It discusses the discovery and characterisation of three proteins, DMI3, CIP73 and IPD3. DMI3 is a CCaMKlike kinase which acts as a master switch for both rhizobial and mycorrhyzal symbioses. IPD3 is a downstream phosphorylation target of DMI3, specific to the nodulation pathway. Its function is not fully elucidated, however is required for correct infection thread formation, and development of functional nodules. CIP73 is also a downstream target of DMI3, however its function is not known. Cip73 mutants show impared nodule development, and reduced rhizobial infection, indicating that CIP73 is required for nodule organogenesis. The discovery of these three proteins represents a significant advance in the elucidation of the molecular mechanisms of rhizobial nodulation, and furthers the goal of harnessing this sustainable source of fixed nitrogen for agricultural purposes.

\section{Introduction}

Legumes have long been known to enter into symbiotic associations with specific rhizobial bacteria, for the purpose of obtaining symbiont-generated nitrogen sources. The interaction between the symbiont and host is highly specific, and involves a complex bidirectional signalling pathway (Lerouge et al. 1990). Flavonoids and isoflavonoids are released by the roots of legumes, which are detected by specific rhizobia. In reply, these release lipochitooligosaccharide molecules termed Nod factors. These Nod factors are perceived by legumes using both nodulation-specific (Nod) and the pathway common to both mycorrhization and nodulation, general symbiosis (Sym), signal transduction pathways, effecting the morphological, biochemical and transcriptomic changes required to allow symbiosis (Ané et al. 2002; Catoira et al. 2000; Lerouge et al. 1990). The upsteam elements of these pathways is shown graphically in Figure 1 
below. The precise elucidation of these signal transduction pathways is crucial to understanding the mechanisms of nodulation, and to any possible future application of this knowledge to crop science and biotechnology. This essay will summarise a number of important studies which have identified elements of these pathways.

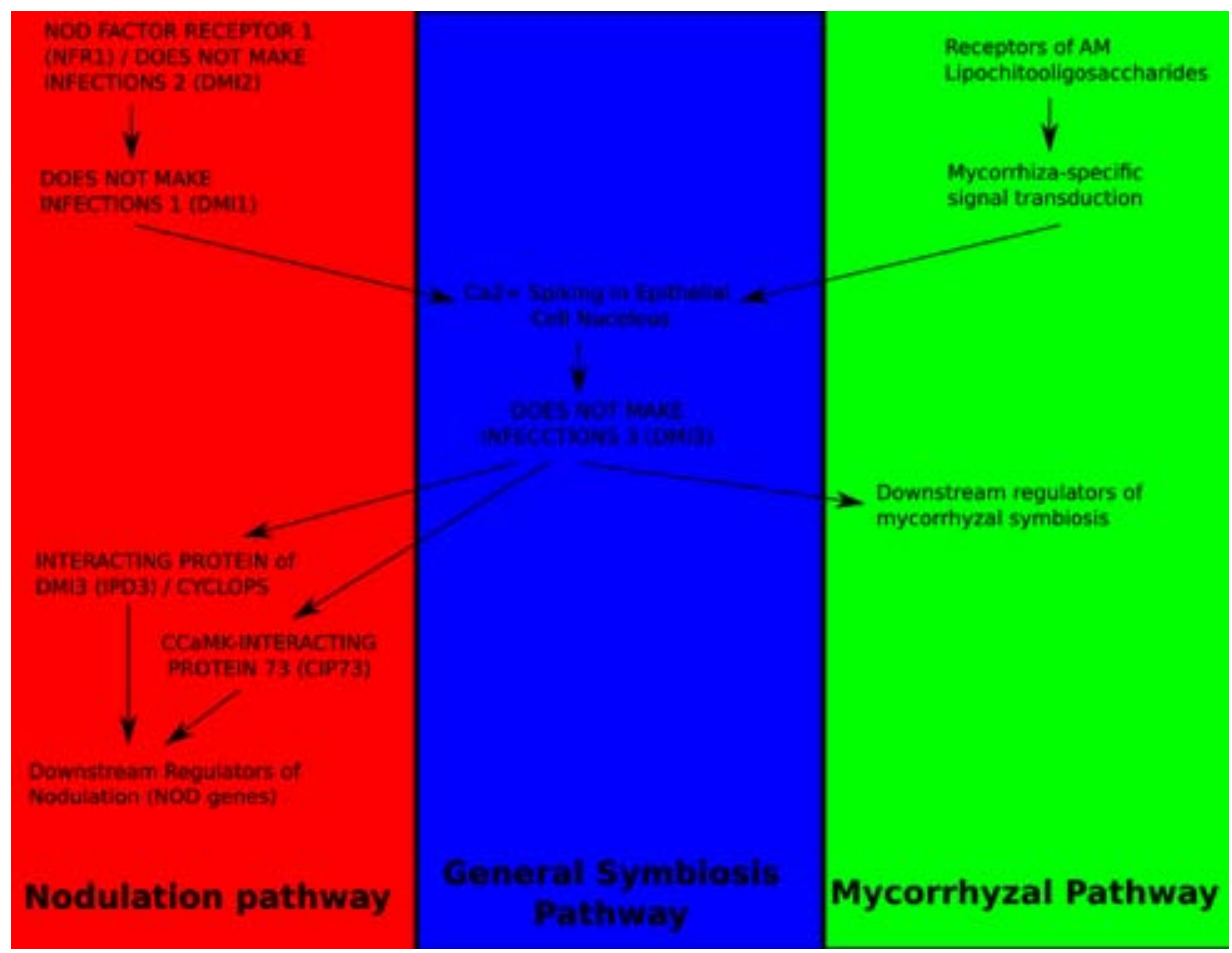

Figure 1: Upstream components of the sym and nod pathways.

The pathways described here are still putative. While the relative ordering of regulatory elements has been at least partially elucidated, no full signalling pathway has been published.

\section{The DMI3 $\mathrm{Ca}^{2+}$ and Camodulin-dependent protein Kinase}

A study by Gleason et. al. (2006) investigated the structure and function of M. truncatula DMI3, key regulator of the Nod and Sym signal transduction pathways. The DMI3 protein is a $\mathrm{Ca}^{2+} /$ Camodulin-dependent protein Kinase (CCaMK), which is activated by $\mathrm{Ca}^{2+}$ and/or camodulin (CaM) binding and phosphorylates specific substrates including itself, signalling the reception of Nod factors to downstream regulatory proteins (Lévy et al. 2004). DMI3 is 
a 523aa protein, which consists of (from $\mathrm{N}$ - to C-terminus) a serine/threonine protein kinase, overlapping CaM binding and autophosphoylation domains and three $\mathrm{EF}$ hand $\mathrm{Ca}^{2+}$ binding domains.

Gleason and colleagues created a series of mutants which lacked various domains of the DMI3 protein, and point mutations which altered the autophosphorylation site. Deletion mutants lacking the autoinhibition domain cannot bind CaM, and constitutively phosphorylate substrates and were termed autoactivated. Autoactivated mutants could not complement dmi3 mutants to allow establishment of symbionts. Point mutations in the autophosphorylation site showed similar phenotypes to autoactivated mutants, however these point mutations complemented dmi3 mutants, and allowed establishment of symbionts.

Gleason and colleagues also investigated the ability of autoactivated mutants of DMI3 to complement other Nod and Sym pathway mutants. Autoactivated DMI3 does not complement GRAS transcription factor mutants nsp2-1 and nsp1-2, indicating that NSP2 and NSP1 probably function downstream of DMI3, and supporting previous studies (Catoira et al. 2000; Kalo 2005; Smit et al. 2005). These authors also observed that autoactivated mutants of DMI3 can complement loss of function mutants in the putative receptor-like kinase DMI2 and the putative cation channel DMI1, indicating that these proteins must function upstream of the DMI3 protein, as suggested by previous results (Catoira et al. 2000; Endre et al. 2002; Ané et al. 2004). Additionally, these authors have shown that expression of Nod factor-induced (NOD) genes becomes constitutive in autoactivated mutants. Expression of a Nod factor-induced GUS expression construct was used to evaluate NOD gene expression; mutants that showed constitutive substrate phosphorylation also showed constitutive GUS expression. This result lends further evidence to the propositions that DMI3 is the master regulator of the nodulation signalling pathway.

Gleason et al. (2006) illustrate the role of each domain in the function of DMI3, and shed light on the regulatory mechanism of this crucial step in the Nod and Sym signal transduction pathways. In wild type $M$. truncatula, $\mathrm{Ca}^{2+}$-induced autophosphorylation is thought to allow binding of CaM to the CaM binding domain, alleviating autoinhibition and allowing substrate phosphorylation. Gleason et al. (2006) demonstrate that removal of the autoinhibition domain causes spontaneous nodule formation in the absence of rhizobia, indicating that this domain negatively regulates DMI3 activity and nodule formation. Gleason et al. (2006) interpret their results in the context of the partially elucidated Nod and Sym signal transduction pathways. These authors propose that DMI3 functions downstream of the DMIl and DMI2 proteins, and regulates expression of NOD genes, indicating it is an upstream regulator of these genes and ultimately 
The ANU Undergraduate Research Journal

of nodulation. This functional characterisation of the DMI3 upstream regulator allowed subsequent studies to elucidate the existence and function of elements downstream of DMI3.

\section{Recent advances in elucidation of the Sym and Nod signal transduction pathways}

\section{Identification of the interacting protein of DMI3 (IPD3) protein}

A study by Messinese et al. (2007) has identified a novel protein which interacts with DMI3 in M. truncatula. Using a yeast two-hybrid $(\mathrm{Y} 2 \mathrm{H})$ system, these authors identified a novel protein, which interacts with DMI3 in vitro and in planta. The IPD3 protein is reported by these authors as functionally redundant for the formation of rhizobial nodules.

Messinese et al. (2007) used a Y2H system to identify the IPD3 protein from a cDNA library from $M$. truncatula roots. A full coding sequence of the gene was obtained, and putative domains were identified. The IPD3 protein was identified as a coiled-coil containing protein, and was mapped to a BAC corresponding to the short arm of $M$. truncatula chromosome 5. Putative orthologs were identified in several moncots and dicots, including L. japonicus but not in Arabidopsis, indicating it is a conserved locus.

A quantitative PCR (qPCR) experiment showed preferential expression of IPD3 and DMI3 to root tissue, and split YFP bimolecular fluorescence complementation studies showed co-localisation of DMI3 and IPD3 to the nuclei of these cells. These results confirm those previously obtained for DMI3 (Gleason et al. 2006). A subsequent mutagenic study of L. japonicus by Yano et al. (2008) identified the CYCLOPS protein, an ortholog of IPD3. These authors show that CYCLOPS is highly structurally and sequentially similar to IPD3, and that it is expressed and localised in the nucleus, similarly to DMI3 and IPD3.

RNAi knock-down caused significant reductions in the qPCR abundance of $I P D 3$ transcripts in transgenic plants, however did not cause any significant change in nodule morphogenesis or rhizobial infection phenotypes. Messinese et al. (2007) suggest that this indicates that either the IPD3 protein is specific to the arbuscule formation pathway (which was not investigated), or that the IPD3 protein is functionally redundant, despite finding no candidate homologs, as nodulation still occurs at low IPD 3 transcript levels. These statements appears to have been disproven by a subsequent study by Horváth et al. (2011), discussed 
in detail below. Additionally, CYCLOPS mutants had phenotypes deficient in formation of functional arbuscules and nodules, but not nodule organogenesis itself (Yano et al. 2008).

Messinese et al. (2007) identify and partially characterise a novel protein that interacts in vivo with the DMI3 protein of $M$. truncatula. This investigation has made important headway in elucidating the signal transduction pathway downstream of CCaMK/DMI3 in $M$. truncatula. However, this paper does not fully characterise the IPD3 protein, nor does this investigation record comprehensive phenotypes of IPD3 mutants, although this is listed as a future priority of the authors.

\section{Further characterisation of the interacting protein of DMI3 (IPD3) protein}

A recent investigation by Horváth et al. (2011) characterises the IPD3 protein identified by Messinese et al. (2007) in a more rigorous manner. These authors identified IPD3 from a transposon-tagged insertion mutagenised $M$. truncatula population, and characterise this mutant, thus inferring the function of IPD3. They find that the primary function of IPD3 is in correct infection thread (IT) formation, and confirm previous mapping, localisation and structural studies and the action of IPD3 as a downstream phosphorylation target of DMI3 (Messinese et al. 2007).

Horváth et al. (2011) report novel results regarding the in planta function of IPD3. Two IPD3 mutants, ipd3-1 and ipd3-2, showed IT formation defective to various degrees; nodules which formed in the presence of rhizobia did not contain bacteroids, and showed no nitrogenase activity. Differences in cellular structure of ITs were also observed. These mutants were complemented with IPD3, both using native and constitutive promoters. Constitutive IPD3 expression did not complement ipd3 mutants, forming non-functional nodules. Native IPD3 expression fully complemented both ipd3 mutants. Ipd3 mutants were also fully complemented with the L. japonicas CYCLOPS ortholog under its native promoter (Yano et al. 2008). The phenotype of IPD3 mutants with regard to arbuscule formation was also investigated, showing that these mutants were defective in the formation of arbuscules. These results also indicate that native temporal specificity in the expression of IPD3 is required for correct function

Ipd3 mutants did not show Nod factor-inducible expression of NOD genes, indicating that IPD3 is upstream of such genes. $\mathrm{A} \mathrm{Ca}^{2+}$ spiking signal identical to that of wild type plants indicates that the IPD3 mutant is downstream of this signal. In the context of previous work on IPD3 (Messinese et al. 2007), this 
places the IPD3 protein between DMI3 and the expression of Nod factor-induced genes. The observation that both arbuscule and nodule formation are affected by ipd 3 mutants places IPD3 in the Sym pathway common to both symbioses.

Horváth et al. (2011) communicate a study which characterises the IPD3 protein. These authors find that IPD3 is a regulatory element downstream of DMI3, but upstream of NOD genes. IPD3 is observed to be crucial to the correct formation of ITs and arbuscules, placing it in the Sym pathway common to both rhizobial and mycorrhizal symbioses.

\section{The CIP73 protein, a novel CCaMK phosphorylation target}

Kang et al. (2011) present a study that identified and characterised a novel protein, which acts as a phosphorylation target for the CCaMK protein of Lotus japonicus. These authors identified a protein that interacted with DMI3 in $\mathrm{Y} 2 \mathrm{H}$ studies, and conducted several further analyses. They determined that CIP73 acts downstream of the DMI3 protein and is part of the Nod pathway.

These authors conducted a $\mathrm{Y} 2 \mathrm{H}$ experiment to determine protein-protein interactions of the serine/threonine protein kinase domain and proteins encoded by an L. japonicus root cDNA library, as a screen for proteins which interact with the kinase domain of CCaMK. Further protein-protein interaction studies showed that in vivo and in planta full length CIP73 interacts with full length CCaMK. The full cDNA sequence was obtained and bioinformatic studies revealed that the protein contained an N-terminal domain with similarity to Xenopus Scythe ubiquitin, and a C-terminal nuclear localisation signal. Orthologous genes were discovered in several plant genomes, including $A$. thaliana and M. truncatula.

Kang and colleagues also investigated the expression and localisation of CIP73. Using qualitative RT-PCR, CIP73 was found to be expressed at higher levels in roots compared to all other tissues. CIP73-GFP gene fusions were found to be localised to the nucleus of epidermal cells of L. japonicus. In vivo studies used to characterise the interaction between CIP73 and CCaMK found that the $\mathrm{N}$-terminus of CIP73 is phosphorylated by CCaMK in yeast. Autophosphorylation of CCaMK was increased by addition of $\mathrm{Ca}^{2+}$, however phosphorylation of CIP73 was only increased in the presence of CaM, a phosphorylation pattern which matches that of CYCLOPS (Yano et al. 2008).

Using RNAi, Kang and colleagues show that the CIP73 protein is required for correct Rhizobium-induced nodule formation, but is not required for arbuscule formation. On inoculation of CIP73 RNAi knockdown roots with Mesorhizobium loti, significantly fewer nodules formed, and observation of nodules stained for $\beta$-galactosidase activity showed that significantly fewer rhizobia reached nodule 
cells. Kang and colleagues interpret this observation to indicate the CIP73 protein controls nodule organogenesis, not rhizobial infection. A similar investigation of arbuscule formation after inoculation of CIP73 RNAi transformants with the mycorrhizal fungus Glomus intraradices found typical arbuscule formation, indicating that CIP73 is specific to the Nod signal transduction pathway.

Kang and colleagues present a comprehensive study, which identifies and characterises a novel protein that is a phosphorylation target for CCaMK in L. japonicus. CIP73 showed expression, localisation and DMI3 interaction characteristics similar to those described in other CCaMK phosphorylation targets (Horváth et al. 2011; Messinese et al. 2007). The CIP73 protein was determined to act downstream of the DMI3 protein. RNAi mutants demonstrate that CIP73 is required for Rhizobium-induced nodule formation, but is not required for arbuscule formation, and therefore is part of the Nod pathway. However, these authors do not propose any mechanism of action for this novel protein.

\section{Conclusion}

This essay has discussed three recent investigations that elucidate and characterise two novel regulatory proteins downstream of the master regulator of the Sym and Nod pathways. IPD3 and its orthologs are regulators crucial to the establishment of symbioses with rhizobia and arbuscular mycorrhizae, but not to organogenesis. The CIP73 protein is required for correct nodule formation, but not for arbuscule formation. While these results were obtained in M. truncatula and L. japonicus, previously observed similarity between $L$. japonicus, M. truncatula and other legumes in several elements of Sym and Nod pathway (Horváth et al. 2011; Sieberer et al. 2009; Yano et al. 2008) suggests that these results may be generalised to most legumes.

Through these and other similar studies we have the beginnings of an outline of regulatory networks involved in nodule formation and rhizobial symbiosis. However, we are far from fully elucidating the full Nod and Sym pathways; nor have we fully characterised the molecular function of discovered elements of these pathways. Given the high cost of nitrogenous fertilisers in a world facing increasing energy costs and food demand, there is keen interest in the application of these discoveries to non-legumes, aiming to impart the symbiontgenerated nitrogen sources to these crops. To be able to put knowledge of rhizobial symbiosis obtained thus far to practical use in these areas, a clearer understanding of the regulation of nodulation is required. Work on elucidation of these pathways is ongoing, and represents an important line of future investigation in plant and crop science. 


\section{References}

Ané, J.-M., Lévy, J., Thoquet, P., Kulikova, O., de Billy, F., Penmetsa, V., Kim, D.J., Debellé, F., Rosenberg, C., Cook, D.R., Bisseling, T., Huguet, T., Dénarié, J., 2002. Genetic and cytogenetic mapping of DMI1,DMI2, and DMI3 genes of Medicago truncatula involved in Nod factor transduction, nodulation, and mycorrhization. Molecular Plant-Microbe Interactions 15: 1108-1118.

Ané, J.-M., Kiss, G.B., Riely, B.K., Penmetsa, R.V., Oldroyd, G.E.D., Ayax, C., Lévy, J., Debellé, F., Baek, J.-M., Kalo, P., Rosenberg, C., Roe, B.A., Long, S.R., Dénarié, J., Cook, D.R., 2004. Medicago truncatula DMIl required for bacterial and fungal symbioses in legumes. Science, 303: 1364-1367.

Catoira, R., Galera, C., de Billy, F., Penmetsa, R.V., Journet, E.-P., Maillet, F., Rosenberg, C., Cook, D., Gough, C., Dénarié, J., 2000. Four genes of Medicago truncatula controlling components of a Nod factor transduction pathway. The Plant Cell 12: 1647 -1666.

Endre, G., Kereszt, A., Kevei, Z., Mihacea, S., Kaló, P., Kiss, G.B., 2002. A receptor kinase gene regulating symbiotic nodule development. Nature, 417: 962-966. 2002.

Gleason, C., Chaudhuri, S., Yang, T., Munoz, A., Poovaiah, B.W., Oldroyd, G.E.D., 2006. Nodulation independent of rhizobia induced by a calciumactivated kinase lacking autoinhibition. Nature, 441:1149-1152.

Horváth, B., Yeun, L.H., Domonkos, Á., Halász, G., Gobbato, E., Ayaydin, F., Miró, K., Hirsch, S., Sun, J., Tadege, M., Ratet, P., Mysore, K.S., Ané, J.-M., Oldroyd, G.E.D., Kaló, P., 2011. Medicago truncatula IPD3 is a member of the common symbiotic signaling pathway required for rhizobial and mycorrhizal symbioses. Molecular Plant-Microbe Interactions, 24: 1345-1358.

Kalo, P., 2005. Nodulation signaling in legumes requires NSP2, a member of the GRAS family of transcriptional regulators. Science, 308: 1786-1789.

Kang, H., Zhu, H., Chu, X., Yang, Z., Yuan, S., Yu, D., Wang, C., Hong, Z., Zhang, Z., 2011. A novel interaction between CCaMK and a protein containing the Scythe_N ubiquitin-like domain in Lotus japonicus. Plant Physiology, 155: $1312-1324$.

Lerouge, P., Roche, P., Faucher, C., Maillet, F., Truchet, G., Promé, J.C., Dénarié, J., 1990. Symbiotic host-specificity of Rhizobium meliloti is determined by a sulphated and acylated glucosamine oligosaccharide signal. Nature, 344: 781-784. 
Lévy, J., Bres, C., Geurts, R., Chalhoub, B., Kulikova, O., Duc, G., Journet, E.-P., Ané, J.-M., Lauber, E., Bisseling, T., Dénarié, J., Rosenberg, C., Debellé, F., 2004. A putative $\mathrm{Ca} 2+$ and calmodulin-dependent protein kinase required for bacterial and fungal symbioses. Science, 303: $1361-1364$.

Messinese, E., Mun, J.-H., Yeun, L.H., Jayaraman, D., Rougé, P., Barre, A., Lougnon, G., Schornack, S., Bono, J.-J., Cook, D.R., Ané, J.-M., 2007. A novel nuclear protein interacts with the symbiotic DMI3 calcium- and calmodulindependent protein kinase of Medicago truncatula. Molecular Plant-Microbe Interactions, 20: 912-921.

Sieberer, B.J., Chabaud, M., Timmers, A.C., Monin, A., Fournier, J., Barker, D.G., 2009. A nuclear-targeted cameleon demonstrates intranuclear $\mathrm{Ca} 2+$ spiking in Medicago truncatula root hairs in response to rhizobial nodulation factors. Plant Physiology, 151: 1197-1206.

Smit, P., Raedts, J., Portyanko, V., Debellé, F., Gough, C., Bisseling, T., Geurts, R., 2005. NSP1 of the GRAS protein family is essential for rhizobial Nod factor-induced transcription. Science, 308: 1789-1791.

Yano, K., Yoshida, S., Müller, J., Singh, S., Banba, M., Vickers, K., Markmann, K., White, C., Schuller, B., Sato, S., Asamizu, E., Tabata, S., Murooka, Y., Perry, J., Wang, T.L., Kawaguchi, M., Imaizumi-Anraku, H., Hayashi, M., Parniske, M., 2008. CYCLOPS, a mediator of symbiotic intracellular accommodation. Proceedings of the National Academy of Sciences of the United States of America, 105: 20540-20545. 